\title{
Communication
}

\section{Appropriateness and Economic Analysis of Conventional Circulating Biomarkers Assessment in Early Breast Cancer: A Real-World Experience from the E.Pic.A Study}

\author{
Roberta Maltoni ${ }^{1, *}+\dagger^{\mathbb{D}}$, William Balzi ${ }^{1,+}+\mathbb{D}$, Tania Rossi ${ }^{2} \mathbb{D}$, Francesco Fabbri ${ }^{2}\left(\mathbb{D}\right.$, Sara Bravaccini ${ }^{2} \mathbb{D}$, \\ Maria Teresa Montella ${ }^{1}$, Ilaria Massa ${ }^{1}{ }^{\mathbb{D}}$, Lucia Bertoni ${ }^{1}$, Fabio Falcini ${ }^{3,4}$ and Mattia Altini ${ }^{5}$
}

\section{check for}

updates

Citation: Maltoni, R.; Balzi, W.; Rossi, T.; Fabbri, F.; Bravaccini, S.; Montella, M.T.; Massa, I.; Bertoni, L.; Falcini, F.; Altini, M. Appropriateness and Economic Analysis of Conventional Circulating Biomarkers Assessment in Early Breast Cancer: A Real-World Experience from the E.Pic.A Study. Curr. Oncol. 2022, 29, 433-438. https://doi.org/10.3390/ curroncol29020039

Received: 8 December 2021 Accepted: 13 January 2022 Published: 18 January 2022

Publisher's Note: MDPI stays neutral with regard to jurisdictional claims in published maps and institutional affiliations.

Copyright: (C) 2022 by the authors. Licensee MDPI, Basel, Switzerland. This article is an open access article distributed under the terms and conditions of the Creative Commons Attribution (CC BY) license (https:// creativecommons.org/licenses/by/ $4.0 /)$.
1 Healthcare Administration, IRCCS, Istituto Romagnolo per lo Studio dei Tumori (IRST) "Dino Amadori", 47014 Meldola, Italy; william.balzi@irst.emr.it (W.B.); mariateresa.montella@irst.emr.it (M.T.M.); ilaria.massa@irst.emr.it (I.M.); lucia.bertoni@irst.emr.it (L.B.)

2 Biosciences Laboratory, IRCCS, Istituto Romagnolo per lo Studio dei Tumori (IRST) "Dino Amadori", 47014 Meldola, Italy; tania.rossi@irst.emr.it (T.R.); francesco.fabbri@irst.emr.it (F.F.); sara.bravaccini@irst.emr.it (S.B.)

3 Cancer Prevention Unit, Local Health Authority, 47121 Forlì, Italy; fabio.falcini@auslromagna.it

4 Romagna Cancer Registry, Romagna Cancer Institute, IRCCS, Istituto Romagnolo per lo Studio dei Tumori (IRST) “Dino Amadori”, 47014 Meldola, Italy

5 Healthcare Administration, Azienda Unità Sanitaria Locale della Romagna, 48121 Ravenna, Italy; mattia.altini@auslromagna.it

* Correspondence: roberta.maltoni@irst.emr.it

+ These authors contributed equally to this work.
Abstract: The risk of relapse for early breast cancer (BC) patients persists even after decades and to date, no specific and sensitive effective circulating biomarker for recurrence prediction has been identified yet. The international guidelines do not recommend the assessment of the serum tumor markers CEA and CA15-3 in the follow-up of asymptomatic early BC patients. In our institute, IRCCS Istituto Romagnolo per lo Studio dei Tumori (IRST) “Dino Amadori”, as part of the E.Pic.A study, which was designed to assess the economic appropriateness of integrated care pathways in early BC, the use of CEA and CA15-3 as circulating tumor biomarkers in early BC patients was evaluated in 1502 patients one year after surgery, from 2015 to 2018, with an overall expense of EUR 51,764. A total of EUR 47,780 (92\%) was used for execution of circulating tumor markers in early BC patients with stage 0 , I and II tumors, neglecting the current guidelines and considered inappropriate by our professional board. We found that no patients with stage I BC experienced relapse in the 365 days after surgery, and in any case examination of the circulating markers CEA and CA15-3 was considered crucial for diagnosis of relapse. Our findings suggest that this inadequacy is a low-value area, supporting the reallocation of economic resources for interventions of a higher value for patients.

Keywords: breast cancer; key performance index (KPI); circulating biomarker; appropriateness; economic resource

\section{Introduction}

It is well known that breast cancer $(\mathrm{BC})$ is a heterogeneous disease, from a biological point of view and natural history. A systemic cancer from its diagnosis can arise in a very aggressive or more indolent manner, but the risk of relapse remains for all patients who undergo surgery for BC, even up to 30-40 years after diagnosis [1]. Consequently, the great clinical need emerges for the ability to use blood circulating markers that can guide physicians on a possible disease relapse.

Despite the efforts made, up to now, we do not have a biomarker with an optimal sensitivity and specificity suitable for predicting a patient's disease relapse. A lot of studies 
have been performed that investigate circulating biomarkers that are useful to predict disease relapse in patients who underwent surgery for early BC [2-6]. For instance, liquid biopsy has the potential to help manage $\mathrm{BC}$ during all stages of disease progression. Circulating Tumor Cells, Extracellular Vesicles, and ctDNA have promise as useful tools in this perspective, describing both spatial and temporal tumor heterogeneity and the sub-clonal evolution of the disease through treatment, and allowing disease and risk of progression to be monitored, aiming at improving personalized medicine [7-9]. Unfortunately, pitfalls arise due to biological and technical reasons, and the type of detection of the investigated biological markers. In particular, some of the them are not exclusively expressed by tumor cells, but also by inflammatory cells, and in other cases, the biomarker has suboptimal accuracy $[10,11]$.

Blood tumor biomarkers such as Carcino-Embryonic Antigen (CEA) and Cancer Antigen 15-3 (CA15-3) are not recommended by American Society of Clinical Oncology (ASCO), European Society for Medical Oncology (ESMO) and Italian Association of Medical Oncology (AIOM) guidelines in the follow-up of early BC patients $[1,12,13]$, in asymptomatic patients. AIOM guidelines in the absence of clinical suspect of relapse do not recommended intensive follow-up (in terms of radiological and blood laboratory examination) during follow-up programs after surgery. Some authors reported in the literature that radiological studies can give false-positive results and increase costs [14-17]. This is also true for serum tumor biomarkers with poor sensitivity and specificity; they should not be recommended as clinical surveillance instruments [18-21].

In a recently published study, it has been reported that in five Italian regions, the percentage of patients undergoing this evaluation in the first year after BC diagnosis appears to be significantly higher than the $20 \%$ benchmark, which was defined as taking into consideration stage IV patients, and other specific conditions in which markers can be indicated [22], systematically neglecting the guidelines.

\section{Findings from the E.Pic.A Study}

In our Institute (IRCCS Istituto Romagnolo per lo Studio dei Tumori (IRST) “Dino Amadori"), the E.Pic.A study, which was specifically designed for BC care, was performed to identify inadequacies in the diagnostic, therapeutic and care pathways with reproducible methods, to evaluate the economic appropriateness of integrated care pathways, to balance the best healthcare possible, and to identify areas of wastage to reallocate the economic resources to high-value activities for patients [23]. The study was approved by the Independent Ethical Committee of the IRST (Reg Sperimentazioni n. 1517, Prot 721/2015; date of approval 17 December 2015). For this purpose, a board of professionals identified seven key performance indexes (KPIs) in the pattern of BC diagnosis and treatment based on the current guidelines from the AIOM [13] and the National Comprehensive Center Network (NCCN) [24]. In this article, preliminary data concerning four KPIs comprised in the E.Pic.A study were shown: KPI-1 (pre-surgery) is defined as the proportion of patients with stage I or II disease who underwent hepatic ultrasound, computed tomography, magnetic resonance imaging, position emission tomography, and bone scan; KPI-2 (post-surgery) includes patients at the same stage who received radiological clinical evaluation within 2 months after breast surgery; KPI-3 (subsequent intervention after mastectomy) is defined as the proportion of patients that were subjected to axillary dissection and/or breast reconstruction within 3 months after mastectomy; KPI-4 (chemotherapy timing) means the proportion of patients that received adjuvant therapy within 60 days after surgery [23]. The KPIs were evaluated in terms of appropriateness and costs, showing that $2798 \mathrm{BC}$ patients received a total of 2156 inappropriate examinations, accounting for EUR 573,510.80.

Based on these findings, we decided to perform further analysis on a cohort of 1502 consecutive $B C$ patients without metastatic disease and other cancers who underwent surgery in the years 2015-2018, assessing the appropriateness of conventional circulating markers (CEA and CA15-3) 365 days after surgery (Table 1). 
Table 1. Distribution of tumors according to staging.

\begin{tabular}{ccccccc}
\hline \multirow{2}{*}{ Stage } & \multicolumn{5}{c}{ Year } & \multirow{2}{*}{$\%$} \\
\cline { 2 - 5 } & $\mathbf{2 0 1 5}$ & $\mathbf{2 0 1 6}$ & $\mathbf{2 0 1 7}$ & $\mathbf{2 0 1 8}$ & Total & \\
\hline 0 & 22 & 23 & 13 & 40 & 98 & 6.5 \\
I & 234 & 196 & 222 & 212 & 864 & 57.5 \\
IIA & 77 & 73 & 85 & 84 & 319 & 21.2 \\
IIB & 37 & 20 & 26 & 36 & 119 & 7.9 \\
IIIA & 13 & 10 & 15 & 21 & 59 & 3.9 \\
IIIB & 1 & 3 & 2 & 5 & 11 & 1 \\
IIIC & 8 & 6 & 11 & 7 & 32 & 2 \\
Total & 392 & $\mathbf{3 3 1}$ & $\mathbf{3 7 4}$ & $\mathbf{4 0 5}$ & $\mathbf{1 5 0 2}$ & $\mathbf{1 0 0}$ \\
\hline
\end{tabular}

The professional board considered the assessment of these conventional circulating markers inappropriate in asymptomatic patients who underwent surgery for stage 0 , I and II tumors (Table 1) as well as for the other KPIs.

The overall cost for CA15-3 and CEA assessment in the 1502 patients in the 365 days following radical surgery was EUR 51,764 (Table 2). This analysis was possible thanks to the access to administrative data. Table 3 shows the overall costs incurred in marker assessment of BC patients with stage 0 , I and II tumors (EUR 47,780).

Based on our findings, $92 \%$ (EUR 47,780) of the overall costs for circulating tumor marker execution (EUR 51,764) were spent in an inappropriate manner in BC patients with stage 0 , I and II tumors.

Considering that the same patients may have performed marker evaluation not only in the first year after surgery but also in the following 5 years and possibly for 10 years after surgery or for the entire life span, the economic impact could not be negligible, especially if translated on a national scale.

Furthermore, the execution of marker detection causes a great deal of emotional stress on patients due to false positive tests (which inevitably generate the execution of instrumental tests) and false tranquility due to a negative outcome when, instead, a metastatic disease may already be present.

Within the 365 days following surgery, 12 out of 1502 patients experienced tumor relapse. The clinical characteristics of the relapsed patients are reported in Table 4 .

Six $(50 \%)$ of the 12 relapsed patients had a diagnosis of triple negative BC (TNBC). Concerning tumor stage, eight patients $(66.7 \%)$ and four patients $(33.3 \%)$ had stage III and stage II BC diagnosis, respectively, but no patients with stage I BC relapsed.

Interestingly, the CA15-3 examination of patient 1 resulted $56.8 \mathrm{KU} / \mathrm{L}$ when liver metastasis was diagnosed, whereas the value was $150 \mathrm{KU} / \mathrm{L}$ and $45 \mathrm{KU} / \mathrm{L}$ before neoadjuvant therapy and after mastectomy, respectively. Patient 3 was diagnosed with axillary relapse after self-examination, with CA15-3 measured after biopsy with a value of $58.5 \mathrm{KU} / \mathrm{L}$. The other relapsed patients displayed CA15-3 values within the normal range $(0-33 \mathrm{KU} / \mathrm{L})$, and the diagnosis of relapse was possible thanks to self-examination or other instrumental assessment. For instance, after self-examination, four patients were diagnosed with lymph node relapse (two cases) and skin relapse (two cases). One patient was diagnosed with skin relapse during routine mammary ultrasound, and two patients with positive axilla at surgery performed a basal bone scan with evidence of bone metastasis. Two patients that underwent abdominal ultrasound were diagnosed with liver metastasis, and one patient was diagnosed with brain metastasis after magnetic resonance imaging following symptoms occurrence such as vertigo and vomit.

Hence, despite the small case series, we found that none of the 12 relapsed patients had a diagnosis of tumor recurrence following increased values of CEA and CA15-3 and downstream instrumental exams. 
Table 2. Monitoring of CEA and CA15-3 execution in the 365 days following radical surgery.

\begin{tabular}{cccccccccc}
\hline Year (Patients) & \multicolumn{2}{c}{2015 (392) } & \multicolumn{2}{c}{$\mathbf{2 0 1 6}(\mathbf{3 3 1}$} & \multicolumn{2}{c}{ 2017 (374) } & \multicolumn{2}{c}{ 2018 (405) } & Total (1502) \\
\hline Marker type & CA15-3 & CEA & CA15-3 & CEA & CA15-3 & CEA & CA15-3 & CEA & CA15-3 or CEA \\
No. of exams & 486 & 352 & 464 & 156 & 641 & 158 & 656 & 161 & 3074 \\
Total amount EUR & 9234 EUR & 3872 EUR & 8797 EUR & 1709 EUR & 12,179 EUR & 1738 EUR & 12,464 EUR & 1771 EUR & 51,764 EUR \\
No. of patients with exams & 288 & 222 & 263 & 115 & 328 & 126 & 334 & 122 & 1222 \\
\% of patients with exams & 73 & 57 & 79 & 35 & 88 & 34 & 82 & 30 & 81 \\
\hline
\end{tabular}

CEA: Carcino-Embryonic Antigen; CA15-3: Cancer Antigen 15-3; EUR: Euro.

Table 3. Monitoring of CEA and CA15-3 assessment in the 365 days following radical surgery, for patients with stage 0 , I, IIA and IIB tumors.

\begin{tabular}{|c|c|c|c|c|c|c|c|c|c|}
\hline \multicolumn{10}{|c|}{ Tumor Stage 0} \\
\hline Year (patients) & \multicolumn{2}{|c|}{$2015(22)$} & \multicolumn{2}{|c|}{$2016(23)$} & \multicolumn{2}{|c|}{$2017(13)$} & \multicolumn{2}{|c|}{$2018(40)$} & Total (98) \\
\hline Marker type & CA15-3 & CEA & CA15-3 & CEA & CA15-3 & CEA & CA15-3 & CEA & CA15-3 or CEA \\
\hline No. of exams & 12 & 10 & 11 & 7 & 5 & 3 & 16 & 9 & 73 \\
\hline Total amount EUR & 228 EUR & 110 EUR & 209 EUR & 77 EUR & 95 EUR & 33 EUR & 304 EUR & 99 EUR & 1155 EUR \\
\hline No. of patients with exams & 9 & 7 & 8 & 5 & 4 & 2 & 10 & 5 & 34 \\
\hline$\%$ of patients with exams & 41 & 32 & 35 & 22 & 31 & 15 & 25 & 13 & 35 \\
\hline \multicolumn{10}{|c|}{ Tumor Stage I } \\
\hline Year (patients) & \multicolumn{2}{|c|}{$2015(234)$} & \multicolumn{2}{|c|}{$2016(196)$} & \multicolumn{2}{|c|}{$2017(222)$} & \multicolumn{2}{|c|}{$2018(212)$} & Total (864) \\
\hline Marker type & CA15-3 & CEA & CA15-3 & CEA & CA15-3 & CEA & CA15-3 & CEA & CA15-3 or CEA \\
\hline No. of exams & 316 & 229 & 292 & 99 & 403 & 92 & 359 & 65 & 1855 \\
\hline Total amount EUR & 6004 EUR & 2519 EUR & 5542 EUR & 1089 EUR & 7657 EUR & 1012 EUR & 6821 EUR & 715 EUR & 31,359 EUR \\
\hline No. of patients with exams & 189 & 144 & 165 & 71 & 206 & 73 & 189 & 55 & 750 \\
\hline$\%$ of patient with exams & 81 & 62 & 84 & 36 & 93 & 33 & 89 & 26 & 87 \\
\hline \multicolumn{10}{|c|}{ Tumor Stage IIA } \\
\hline Year (patients) & \multicolumn{2}{|c|}{$2015(77)$} & \multicolumn{2}{|c|}{$2016(73)$} & \multicolumn{2}{|c|}{$2017(85)$} & \multicolumn{2}{|c|}{$2018(84)$} & Total (319) \\
\hline Marker type & CA15-3 & CEA & CA15-3 & CEA & CA15-3 & CEA & CA15-3 & CEA & CA15-3 or CEA \\
\hline No. of exams & 92 & 68 & 104 & 31 & 131 & 39 & 151 & 52 & 668 \\
\hline Total amount EUR & 1748 EUR & 748 EUR & 1976 EUR & 341 EUR & 2489 EUR & 429 EUR & 2869 EUR & 572 EUR & 11,172 EUR \\
\hline No. of patients with exams & 49 & 44 & 59 & 26 & 69 & 32 & 79 & 37 & 261 \\
\hline$\%$ of patient with exams & 64 & 57 & 81 & 36 & 81 & 38 & 94 & 44 & 82 \\
\hline \multicolumn{10}{|c|}{ Tumor Stage IIB } \\
\hline Year (patients) & \multicolumn{2}{|c|}{$2015(37)$} & \multicolumn{2}{|c|}{$2016(20)$} & \multicolumn{2}{|c|}{$2017(26)$} & \multicolumn{2}{|c|}{$2018(36)$} & Total (119) \\
\hline Marker type & CA15-3 & CEA & CA15-3 & CEA & CA15-3 & CEA & CA15-3 & CEA & CA15-3 or CEA \\
\hline No. of exams & 50 & 36 & 29 & 5 & 42 & 9 & 58 & 13 & 242 \\
\hline Total amount EUR & 950 EUR & 396 EUR & 551 EUR & 55 EUR & 798 EUR & 99 EUR & 1102 EUR & 143 EUR & 4094 EUR \\
\hline No. of patients with exams & 30 & 23 & 18 & 4 & 23 & 9 & 27 & 10 & 98 \\
\hline$\%$ of patient with exams & 81 & 62 & 90 & 20 & 88 & 35 & 75 & 28 & 82 \\
\hline \multicolumn{9}{|c|}{ Total amount for stage 0 , I, IIA and IIB } & 47,780 EUR \\
\hline
\end{tabular}

CEA: Carcino-Embryonic Antigen; CA15-3: Cancer Antigen 15-3; EUR: Euro.

Table 4. Clinicopathological features of patients who underwent relapse within the 365 days after surgery. CEA was considered normal with values $<5 \mu \mathrm{g} / \mathrm{L}$. CA15-3 was considered normal with values $<33 \mathrm{KU} / \mathrm{L}$.

\begin{tabular}{ccccccccccc}
\hline $\begin{array}{c}\text { Patient } \\
\text { Number }\end{array}$ & Stage & $\begin{array}{c}\text { Age At } \\
\text { Surgery }\end{array}$ & $\begin{array}{c}\text { Surgery } \\
\text { Type }\end{array}$ & ER & PgR & Ki67/Mib1 & HER2 & Relapse Site & $\begin{array}{c}\text { CEA } \\
\text { (ug/L) }\end{array}$ & $\begin{array}{c}\text { CA15-3 } \\
\text { (KU/L) }\end{array}$ \\
\hline $\mathbf{1}$ & IIIA & 72 & M & $20 \%$ & $10 \%$ & $20 \%$ & 0 & Liver & NP & 56.8 \\
$\mathbf{2}$ & IIIC & 53 & Q & $0 \%$ & $0 \%$ & $67 \%$ & 0 & Axillary lymph node & Normal & Normal \\
$\mathbf{3}$ & IIA & 79 & M & $0 \%$ & $0 \%$ & $80 \%$ & 0 & Axillary lymph node & NP & 58.5 \\
$\mathbf{4}$ & IIA & 64 & M & $0 \%$ & $0 \%$ & $70 \%$ & 0 & Skin & Normal & Normal \\
$\mathbf{5}$ & IIIC & 49 & Q & $100 \%$ & $100 \%$ & $5 \%$ & 0 & Bones & NP & Normal \\
$\mathbf{6}$ & IIIB & 87 & M & $90 \%$ & $35 \%$ & $21 \%$ & 0 & Skin & NP & Normal \\
$\mathbf{7}$ & IIB & 51 & M & $0 \%$ & $0 \%$ & $75 \%$ & 0 & Brain & NP & Normal \\
$\mathbf{8}$ & IIIC & 72 & M & $100 \%$ & $0 \%$ & $25 \%$ & +++ & Liver & NP & Normal \\
$\mathbf{9}$ & IIB & 89 & Q & $50 \%$ & $10 \%$ & $40 \%$ & 0 & Bones & NP & Normal \\
$\mathbf{1 0}$ & IIIC & 84 & M & $0 \%$ & $0 \%$ & $30 \%$ & 0 & Axillary extension & NP & Normal \\
$\mathbf{1 1}$ & IIIA & 47 & M & $10 \%$ & $10 \%$ & $40 \%$ & 0 & Skin & NP & Normal \\
$\mathbf{1 2}$ & IIIB & 80 & M & $0 \%$ & $0 \%$ & $35 \%$ & 0 & Liver & NP & NP \\
\hline
\end{tabular}




\section{Conclusions}

In our real-world experience, we found that no patients with stage I BC experienced relapse in the 365 days after surgery, and in any case, examination of the circulating markers CEA and CA15-3 was considered crucial for diagnosis of relapse.

Our findings identify an area of low-value use of resources that could be better reallocated to interventions with a higher value for the patient. Hence, sharing these results with physicians is noteworthy to redirect the current clinical practice to an improved compliance with the guidelines drafted by scientific associations.

Author Contributions: Conceptualization, R.M., F.F. (Fabio Falcini) and M.A.; formal analysis, R.M. and W.B.; data curation, R.M., W.B. and T.R.; writing-original draft preparation, R.M., T.R., F.F. (Francesco Fabbri), S.B. and I.M.; writing-review and editing, R.M., W.B., T.R., I.M. and L.B.; supervision, R.M., M.T.M., F.F. (Fabio Falcini) and M.A. All authors have read and agreed to the published version of the manuscript.

Funding: This work was supported by a public-private partnership between Roche and IRST IRCCS. The funding source had no role in the study design nor in the writing of the manuscript.

Institutional Review Board Statement: The study was approved by the Independent Ethical Committee of the IRST (Reg Sperimentazioni n. 1517, Prot 721/2015; date of approval 17 December 2015).

Informed Consent Statement: The study was approved by the Independent Ethical Committee of the IRST (Reg Sperimentazioni n. 1517, Prot 721/2015). Obtaining informed consent forms was waived in accordance with the current legislation. Written informed consent has been obtained from the patient(s) to publish this paper.

Data Availability Statement: The data presented in this study are available on request from the corresponding author.

Conflicts of Interest: The authors declare no conflict of interest.

\section{References}

1. Cardoso, F.; Kyriakides, S.; Ohno, S.; Penault-Llorca, F.; Poortmans, P.; Rubio, I.T.; Zackrisson, S.; Senkus, E. Early breast cancer: ESMO Clinical Practice Guidelines for diagnosis, treatment and follow-up. Ann. Oncol. 2019, 30, 1194-1220. [CrossRef]

2. Maltoni, R.; Casadio, V.; Ravaioli, S.; Foca, F.; Tumedei, M.M.; Salvi, S.; Martignano, F.; Calistri, D.; Rocca, A.; Schirone, A.; et al. Cell-free DNA detected by "liquid biopsy" as a potential prognostic biomarker in early breast cancer. Oncotarget 2017, 8 , 16642-16649. [CrossRef]

3. Maltoni, R.; Palleschi, M.; Ravaioli, S.; Tumedei, M.M.; Rocca, A.; Melegari, E.; Altini, M.; Puccetti, M.; Manunta, S.; Bravaccini, S. Cell-Free DNA Variant Sequencing Using CTC-Depleted Blood for Comprehensive Liquid Biopsy Testing in Metastatic Breast Cancer. Cell Transpl. 2020, 29, 096368972092505. [CrossRef] [PubMed]

4. Maltoni, R.; Fici, P.; Amadori, D.; Gallerani, G.; Cocchi, C.; Zoli, M.; Rocca, A.; Cecconetto, L.; Folli, S.; Scarpi, E.; et al. Circulating tumor cells in early breast cancer: A connection with vascular invasion. Cancer Lett. 2015, 367, 43-48. [CrossRef] [PubMed]

5. Granato, A.M.; Frassineti, G.L.; Giovannini, N.; Ballardini, M.; Nanni, O.; Maltoni, R.; Amadori, D.; Volpi, A. Do Serum Angiogenic Growth Factors Provide Additional Information to That of Conventional Markers in Monitoring the Course of Metastatic Breast Cancer? Tumor Biol. 2006, 27, 302-308. [CrossRef]

6. Bronte, G.; Ravaioli, S.; Bravaccini, S. What to look for in cell-free DNA from breast cancer patients. Breast Cancer Res. Treat. 2018, 170, 199-200. [CrossRef] [PubMed]

7. Vasseur, A.; Kiavue, N.; Bidard, F.; Pierga, J.; Cabel, L. Clinical utility of circulating tumor cells: An update. Mol. Oncol. 2020, 15, 1647-1666. [CrossRef]

8. Liu, T.; Hooda, J.; Atkinson, J.M.; Whiteside, T.L.; Oesterreich, S.; Lee, A.V. Exosomes in breast cancer-Mechanisms of Action and Clinical Potential. Mol. Cancer Res. 2021, 19, 935-945. [CrossRef]

9. De Mattos-Arruda, L.; Siravegna, G. How to use liquid biopsies to treat patients with cancer. ESMO Open 2021, 6, 100060. [CrossRef] [PubMed]

10. Fabian, C.J.; Kimler, B.F. Use of biomarkers for breast cancer risk assessment and prevention. J. Steroid Biochem. Mol. Biol. 2007, 106, 31-39. [CrossRef]

11. Volpi, A.; Bravaccini, S.; Medri, L.; Cerasoli, S.; Gaudio, M.; Amadori, D. Usefulness of Immunological Detection of the Human Telomerase Reverse Transcriptase. Anal. Cell. Pathol. 2005, 27, 347-353. [CrossRef]

12. Khatcheressian, J.L.; Hurley, P.; Bantug, E.; Esserman, L.J.; Grunfeld, E.; Halberg, F.; Hantel, A.; Henry, N.L.; Muss, H.B.; Smith, T.J.; et al. Breast Cancer Follow-Up and Management After Primary Treatment: American Society of Clinical Oncology Clinical Practice Guideline Update. J. Clin. Oncol. 2013, 31, 961-965. [CrossRef] [PubMed] 
13. Associazione Italiana di Oncologia Medica (AIOM). Linee Guida Neoplasie Della Mammella Edizione 2020. Available online: https://www.aiom.it/wp-content/uploads/2020/10/2020_LG_AIOM_Neoplasie_Mammella_16022021.pdf (accessed on 8 April 2021).

14. Schreve, R.H.; Terpstra, O.T.; Ausema, L.; Lameris, J.S.; Van Seijen, A.J.; Jeekel, J. Detection of liver metastases. A prospective study comparing liver enzymes, scintigraphy, ultrasonography and computed tomography. Br. J. Surg. 2005, 71, 947-949. [CrossRef]

15. Kemeny, M.M.; Sugarbaker, P.H.; Smith, T.J.; Edwards, B.K.; Shawker, T.; Vermess, M.; Jones, A.E. A Prospective Analysis of Laboratory Tests and Imaging Studies To Detect Hepatic Lesions. Ann. Surg. 1982, 195, 163-167. [CrossRef] [PubMed]

16. Wiener, S.N. An Assessment of Routine Liver Scanning in Patients With Breast Cancer. Arch. Surg. 1978, 113, 126. [CrossRef]

17. Chan, D.W.; Beveridge, R.A.; Muss, H.; Fritsche, H.A.; Hortobagyi, G.; Theriault, R.; Kiang, D.; Kennedy, B.J.; Evelegh, M. Use of Truquant BR radioimmunoassay for early detection of breast cancer recurrence in patients with stage II and stage III disease. $J$. Clin. Oncol. 1997, 15, 2322-2328. [CrossRef] [PubMed]

18. Emens, L.A.; Davidson, N.E. The follow-up of breast cancer. Semin. Oncol. 2003, 30, 338-348. [CrossRef]

19. Nicolini, A.; Tartarelli, G.; Carpi, A.; Metelli, M.R.; Ferrari, P.; Anselmi, L.; Conte, M.; Berti, P.; Miccoli, P. Intensive post-operative follow-up of breast cancer patients with tumour markers: CEA, TPA or CA15.3 vs MCA and MCA-CA15.3 vs CEA-TPA-CA15.3 panel in the early detection of distant metastases. BMC Cancer 2006, 6, 269. [CrossRef] [PubMed]

20. Valenzuela, P.; Mateos, S.; Tello, E.; López-Bueno, M.J.; Garrido, N.; Gaspar, M.J. The contribution of the CEA marker to CA 15.3 in the follow-up of breast cancer. Eur. J. Gynaecol. Oncol. 2003, 24, 60-62.

21. Guadagni, F.; Ferroni, P.; Carlini, S.; Mariotti, S.; Spila, A.; Aloe, S.; D’Alessandro, R.; Carone, M.D.; Cicchetti, A.; Ricciotti, A.; et al. A re-evaluation of carcinoembryonic antigen (CEA) as a serum marker for breast cancer: A prospective longitudinal study. Clin. Cancer Res. 2001, 7, 2357-2362. [PubMed]

22. Guarneri, V.; Pronzato, P.; Bertetto, O.; Roila, F.; Amunni, G.; Bortolami, A.; Tognazzo, S.; Griguolo, G.; Pagano, E.; Stracci, F.; et al. Use of Electronic Administrative Databases to Measure Quality Indicators of Breast Cancer Care: Experience of Five Regional Oncology Networks in Italy. JCO Oncol. Pract. 2020, 16, e211-e220. [CrossRef] [PubMed]

23. Massa, I.; Balzi, W.; Burattini, C.; Gentili, N.; Bucchi, L.; Nanni, O.; Gallegati, D.; Pierini, A.; Amadori, D.; Falcini, F.; et al. The challenge of sustainability in healthcare systems: Frequency and cost of inappropriate patterns of breast cancer care (the E.Pic.A study). Breast 2017, 34, 103-107. [CrossRef] [PubMed]

24. NCCN: NCCN Clinical Practice Guidelines in Oncology-Breast Cancer, Version 1.2019. Available online: https://www.nccn. org/professionals/physician_gls/pdf/breast.pdf (accessed on 8 April 2021). 